\title{
The Origin of the RB1 Imprint
}

\author{
Deniz Kanber ${ }^{1 *}$, Karin Buiting ${ }^{1}$, Christian Roos², Jörg Gromoll ${ }^{3}$, Sabine Kaya ${ }^{1}$, Bernhard Horsthemke ${ }^{1}$, \\ Dietmar Lohmann ${ }^{1}$
}

1 Institut für Humangenetik, Universitätsklinikum Essen, Essen, Germany, 2 Gene Bank of Primates und Abteilung Primatengenetik, Deutsches Primatenzentrum, Leibniz-Institut für Primatenforschung, Göttingen, Germany, 3 Centrum für Reproduktionsmedizin und Andrologie, Universitätsklinikum Münster, Münster, Germany

\begin{abstract}
The human $R B 1$ gene is imprinted due to a differentially methylated $\mathrm{CpG}$ island in intron 2. This CpG island is part of $P P P 1 R 26 P 1$, a truncated retrocopy of PPP1R26, and serves as a promoter for an alternative RB1 transcript. We show here by in silico analyses that the parental PPP1R26 gene is present in the analysed members of Haplorrhini, which comprise Catarrhini (Old World Monkeys, Small apes, Great Apes and Human), Platyrrhini (New World Monkeys) and tarsier, and Strepsirrhini (galago). Interestingly, we detected the retrocopy, PPP1R26P1, in all Anthropoidea (Catarrhini and Platyrrhini) that we studied but not in tarsier or galago. Additional retrocopies are present in human and chimpanzee on chromosome 22, but their distinct composition indicates that they are the result of independent retrotransposition events. Chimpanzee and marmoset have further retrocopies on chromosome 8 and chromosome 4, respectively. To examine the origin of the $R B 1 \mathrm{imprint}$, we compared the methylation patterns of the parental PPP1R26 gene and its retrocopies in different primates (human, chimpanzee, orangutan, rhesus macaque, marmoset and galago). Methylation analysis by deep bisulfite sequencing showed that PPP1R26 is methylated whereas the retrocopy in $R B 1$ intron 2 is differentially methylated in all primates studied. All other retrocopies are fully methylated, except for the additional retrocopy on marmoset chromosome 4, which is also differentially methylated. Using an informative SNP for the methylation analysis in marmoset, we could show that the differential methylation pattern of the retrocopy on chromosome 4 is allele-specific. We conclude that the epigenetic fate of a PPP1R26 retrocopy after integration depends on the DNA sequence and selective forces at the integration site.
\end{abstract}

Citation: Kanber D, Buiting K, Roos C, Gromoll J, Kaya S, et al. (2013) The Origin of the RB1 Imprint. PLoS ONE 8(11): e81502. doi:10.1371/journal.pone 0081502

Editor: Roscoe Stanyon, University of Florence, Italy

Received July 12, 2013; Accepted October 23, 2013; Published November 25, 2013

Copyright: @ 2013 Kanber et al. This is an open-access article distributed under the terms of the Creative Commons Attribution License, which permits unrestricted use, distribution, and reproduction in any medium, provided the original author and source are credited.

Funding: Supported by the Deutsche Forschungsgemeinschaft (DFG, LO 530/7-1). The funder had no role in study design, data collection and analysis, decision to publish, or preparation of the manuscript.

Competing interests: The authors have declared that no competing interests exist.

*E-mail: deniz.kanber@uni-due.de

\section{Introduction}

Retrotransposition describes the process of reversetranscription of an mRNA followed by the insertion into a new genomic location, thus forming a retrocopy of its parental gene [1]. This mechanism provides a source for genome variability and contributes to genome evolution. Retrocopies were misleadingly thought to be "junk DNA" for a long time as they do not have promoter regions and often carry deletions or mutations, but many functional retrocopies have been detected in the past years. Functional retrocopies can be either proteincoding [2] or they can have a regulatory function as they can serve as miRNA decoys, produce siRNAs or function as antisense transcripts [3,4,5]. Mcts2, Nap1/5 and Zrsr1 are imprinted retrocopies located in introns that influence the expression of their respective host genes by transcriptional interference $[6,7,8,9]$. Epigenetically controlled transcriptional interference by retrocopies also takes place on human chromosome 13, where intron 2 of the $R B 1$ gene harbours $P P P 1 R 26 P 1$, a truncated retrocopy of $P P P 1 R 26$. In contrast to the aforementioned retrogenes, a fusion of the retrocopy and the host gene has developed at this locus. PPP1R26P1 is integrated in reverse orientation relative to $R B 1$ and its exon 4 contains a $\mathrm{CpG}$ island that gained promoter activity and imprinted methylation. Moreover, this CpG island harbours a start exon which is spliced onto exon 3 of the $R B 1$ gene leading to skewed $R B 1$ expression [10]. Because mouse $R b 1$ neither contains the retrocopy nor shows imprinted expression, we focused our analyses on primates. We compared the sequences of the homologous retrocopies in the available primate genomes, determined methylation patterns of the included $\mathrm{CpG}$ islands and identified expression of transcripts initiating within these regions to examine the origin of the human $R B 1$ imprint. 


\section{Results}

\section{Primate genomes contain retrocopies of PPP1R26}

A Blat search with the mRNA sequence of the PPP1R26 gene showed that the genomes available from Anthropoidea (human - Homo sapiens, chimpanzee - Pan troglodytes, orangutan - Pongo abelii, rhesus macaque - Macaca mulatta, marmoset - Callithrix jacchus), tarsier (Tarsius syrichta) and galago (Otolemur garnettii) contain this gene with conserved exon-intron organization. We could not determine if the 5'-part of PPP1R26 is conserved in the following species as the genome information available for rhesus macaque, marmoset and galago showed sequencing gaps in the regions corresponding to exon 1 , and the tarsier genome data is incomplete so only part of exon 4 of PPP1R26 was detected. We conclude that PPP1R26 is present in tarsier although only partly detected because of the presence of PPP1R26 in Anthropoidea as well as in galago.

The Blat search revealed PPP1R26P1, a 5'-truncated retrocopy of $P P P 1 R 26$, in intron 2 of the $R B 1$ gene in all Anthropoidea studied (Figure 1). However, two copies of PPP1R26P1 are annotated in the genome database of orangutan in $R B 1$ intron 2 as head-to-tail tandem repeats. This annotation could be incorrect as these two copies represent end sequences of neighbouring sequence contigs with a sequence gap between the contigs. This was supported by the results of our quantitative $P C R$ that showed only one copy of $P P P 1 R 26 P 1$ in orangutan (Figure S1). PPP1R26P1 was found neither in tarsier nor in galago, irrespective of which tool (Blat, blastn or Progressive Mauve) was used (Figure S2). The retrocopies in intron 2 of the RB1 gene in human, chimpanzee, orangutan and rhesus macaque genomes have an Alu repeat insertion of about $300 \mathrm{bp}$ between the regions corresponding to exon 3 and exon 4 of the PPP1R26 gene, but the insertion has no sequence identity to PPP1R26. The marmoset PPP1R26P1 has no Alu insertion but a duplication of about $530 \mathrm{bp}$ in the region corresponding to exon 4 of $P P P 1 R 26$.

On chromosome 22 of human and chimpanzee there are further 4 and 3 retrocopies, respectively. In human, all chromosome 22 retrocopies consist of the complete exons 1, 2, 3 and 4 and have an insertion of about 250 bp between exon 1 and exon 2 of the parental sequences that has $81 \%$ sequence identity to the 5'-region of intron 1 of PPP1R26. In chimpanzee, two of the three chromosome 22 retrocopies show the same intron-derived insertion. Sequence data for the third chromosome 22 retrocopy in the chimpanzee is incomplete in this region because of a sequencing gap that spans sequences corresponding to the 3'-part of exon 1 and the 5'-part of exon 2. The third chimpanzee retrocopy is truncated by $1500 \mathrm{bp}$ at the 3 '-end of exon 4, and one of the other two chimpanzee chromosome 22 retrocopies is truncated at the 5'-end.

Apart from the retrocopies in the $R B 1$ gene and on chromosome 22 in human and chimpanzee, the Blat search revealed additional retrocopies only on chimpanzee chromosome 8 and marmoset chromosome 4. Both consist of complete exons 1, 2, 3 and 4 . The retrocopy on chimpanzee chromosome 8 is located in a gene desert, whereas the retrocopy on marmoset chromosome 4 is located in a region that corresponds to the major histocompatibility complex class I region on human chromosome 6 and harbours several genes, including HLA-E, HLA-L, TRIM39 and RPP21.

\section{PPP1R26 and its retrocopies contain CpG islands}

Human, chimpanzee and orangutan have a large (>300 bp) $\mathrm{CpG}$ island at the promoter region of the PPP1R26 gene (UCSC CpG island track) [11]. Human and orangutan have four additional small $\mathrm{CpG}$ islands in exon 4 of PPP1R26, whereas chimpanzee and marmoset have one small and one large CpG island, and rhesus macaque has two small and one large CpG island in this region. Galago has no $\mathrm{CpG}$ islands in exon 4 (Figure 1). We have not included tarsier to Figure 1 as only part of exon 4 of PPP1R26 was detected and no methylation analysis was conducted (no material was available).

The retrocopy in $R B 1$ intron 2, PPP1R26P1, is 5'-truncated and has no $\mathrm{CpG}$ island in what is left from exon 1. However, the region of PPP1R26P1 that corresponds to exon 4 of the parental PPP1R26 gene has two large CpG islands in all Anthropoidea that we studied. Rhesus macaque has one additional and marmoset has two additional small $\mathrm{CpG}$ islands in this region.

The four retrocopies on human chromosome 22 all have a large $\mathrm{CpG}$ island in the sequence corresponding to exon 1 and no $\mathrm{CpG}$ island in the sequence corresponding to exon 4. The two retrocopies on chimpanzee chromosome 22 containing a full length exon 1 have a CpG island in this region, although the true extent of the $\mathrm{CpG}$ island in one of these retrocopies might be underestimated because of a sequencing gap in the available genome data. Both retrocopies with full length exon 4 have one small $\mathrm{CpG}$ island near the 3'-end.

The retrocopies on chimpanzee chromosome 8 and marmoset chromosome 4 have a large CpG island in exon 1 . The sequences derived from exon 4 of the parental gene show two large $\mathrm{CpG}$ islands in marmoset, and one large and one small $\mathrm{CpG}$ island in chimpanzee. However, as the small $\mathrm{CpG}$ island ( $\mathrm{CpG} 18)$ in the chimpanzee is next to a sequencing gap in the genome database, this $\mathrm{CpG}$ island could in fact be larger than annotated.

\section{CpG islands in PPP1R26 and its retrocopies have individual methylation patterns}

We used deep bisulfite sequencing to study the methylation patterns of the $\mathrm{CpG}$ islands in exon 4 of PPP1R26 and in its retrocopies in primates (human, chimpanzee, rhesus macaque, orangutan, marmoset and galago; Figure 2). Two individual samples were analysed for each primate species, except for galago, for which only one sample was available. Detailed data and the mean methylation levels of each CpG island are shown in Figure 2. In figure 1, a pie chart indicates the average methylation at each $\mathrm{CpG}$ island analysed. In all primates studied, the CpG islands in PPP1R26 exon 4 showed a methylation level of around $90 \%$ (Figure 1 pie charts, Figure 2). There are no $\mathrm{CpG}$ islands annotated in exon 4 in galago, so we studied the methylation of the region that corresponds by location to the $\mathrm{CpG}$ island analysed for PPP1R26.

The large CpG island in the exon 4 region of $P P P 1 R 26 P 1$ showed methylated and unmethylated sequences in all 


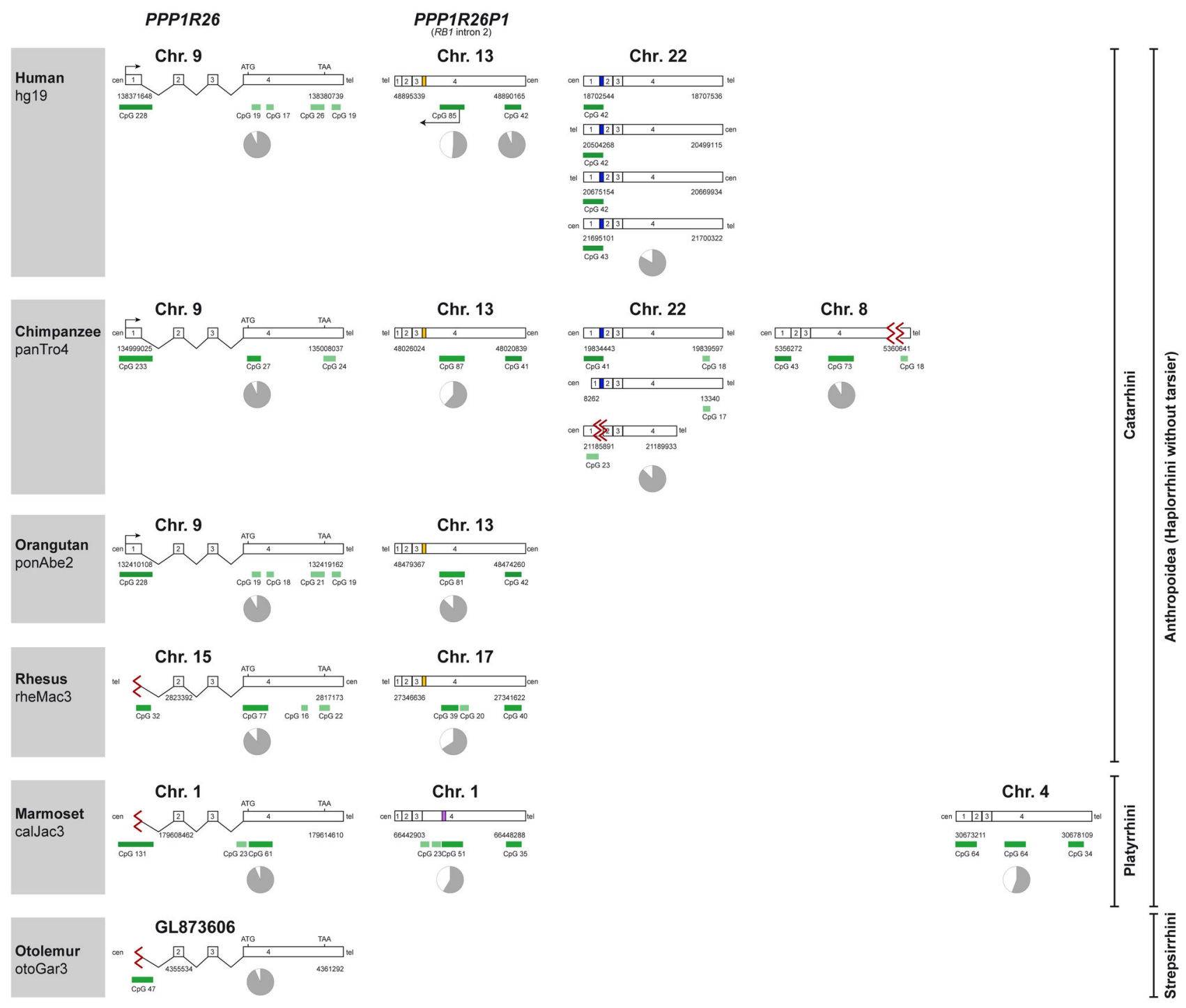

Figure 1. Primate genomes contain retrocopies of PPP1R26. All primates analysed contain the PPP1R26 gene. All Anthropoidea have PPP1R26P1, a 5'-truncated retrocopy of PPP1R26 inserted into intron 2 of the RB1 gene, which is missing in galago (Otolemur garnettii, Strepsirrhini). All Anthropoidea except marmoset have an Alu element (yellow box) inserted in this retrocopy. Apart from the retrocopies in the RB1 gene, the Blat search revealed additional retrocopies on chromosome 22 in human (four retrocopies) and chimpanzee (three retrocopies) and one additional retrocopy on chimpanzee chromosome 8 and marmoset chromosome 4. The pie charts indicate the position of the analysed $\mathrm{CpG}$ islands and their degree of methylation (grey, methylated; white, unmethylated). yellow box, Alu element; purple box, $\sim 550 \mathrm{bp}$ duplication; blue box, $\sim 250 \mathrm{bp}$ intronic sequence of intron 1 of the parental gene; green boxes, $\mathrm{CpG}$ islands (>300 bp dark; $<300 \mathrm{bp}$ light); red sigma sign, gap.

doi: 10.1371/journal.pone.0081502.g001

primates studied (Figure 1 pie charts, Figure 2). The degree of methylation was about $60 \%$ in all species except orangutan, which showed differential methylation only at some CpG sites whereas adjacent regions were highly methylated. Separating the orangutan alleles revealed that one allele is completely methylated at these sites whereas the other allele is $56 \%$ methylated (Figure S3). Previously, we have shown that the methylation at this CpG island is parent-of-origin-specific in human as the paternal allele is unmethylated and the maternal allele is methylated [10]. Analysis of members of a rhesus macaque family heterozygous for a SNP $(G / T)$ in this region revealed that one allele is methylated and the other allele is unmethylated in rhesus as well (Figure $3 \mathrm{~A}$ and $\mathrm{B}$ ). However, the parental origin of the alleles cannot be resolved as all family members are heterozygous. In a marmoset family with an informative SNP $(\mathrm{C} / \mathrm{G})$, average methylation of the two alleles was $72 \%$ and $39 \%$ (Figure $3 \mathrm{C}$ and D). Determination of the parental origin of the alleles is not feasible due to the high 


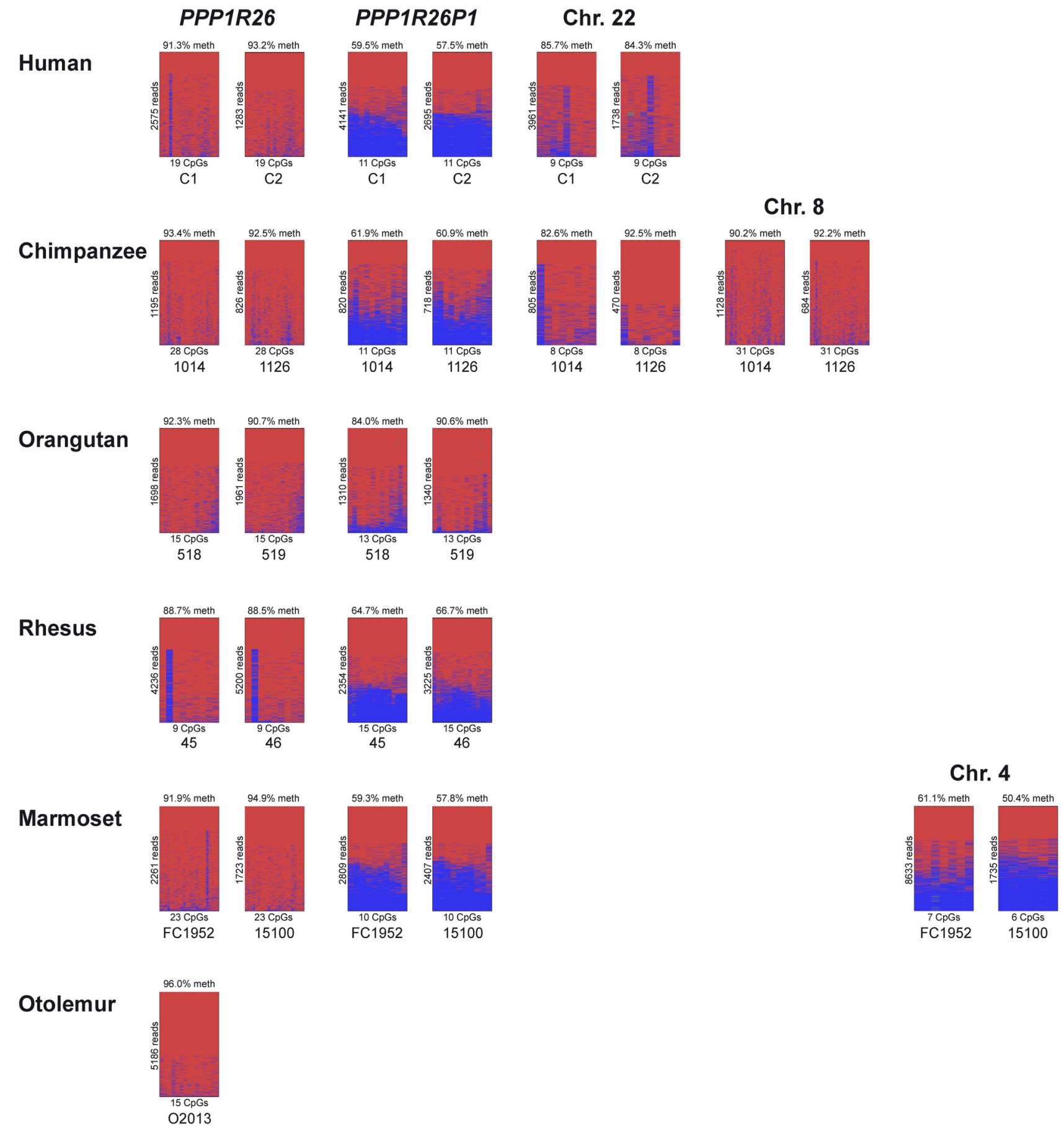

Figure 2. Methylation status of CpG islands in PPP1R26 and its retrocopies. The CpG islands in the 5'-part of exon 4 studied for PPP1R26 and its retrocopies on chromosome 22 and 8 are fully methylated. Only the retrocopy in intron 2 of the RB1 gene (orangutan shows differential methylation only at some CpG sites) and the additional retrocopy on chromosome 4 in marmoset show differential methylation. red, methylated; blue, unmethylated. Blood sample IDs are given under the images (C1, C2 - human; 1014, 1126 - chimpanzee; 518, 519 - orangutan; 45, 46 - rhesus macaque; FC49, 15100 - marmoset; O2013 - galago).

doi: 10.1371/journal.pone.0081502.g002

rates of genetic microchimerism in marmosets [12]. In addition to the differentially methylated $\mathrm{CpG}$ island (CpG 85) that leads to imprinted $R B 1$ expression in human, the region of PPP1R26P1 in Anthropoidea that is derived from exon 4 of the 
A

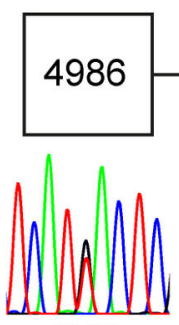

$\mathrm{G} / \mathrm{T}$
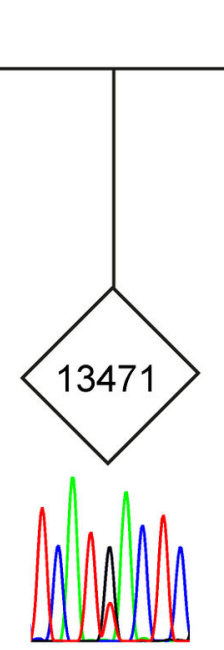

$\mathrm{G} / \mathrm{T}$

C

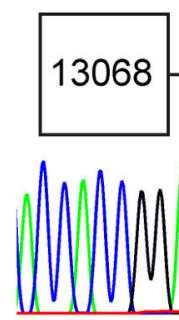

$\mathrm{C} / \mathrm{C}$
9302

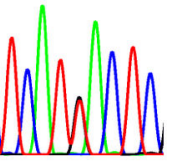

$\mathrm{G} / \mathrm{T}$

B
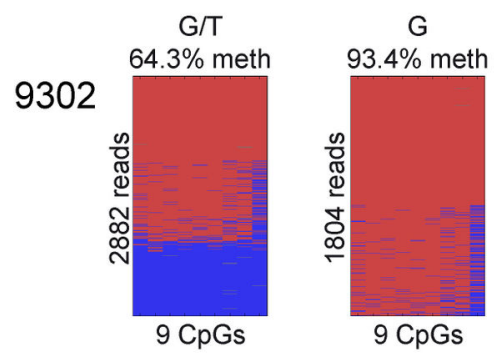

$\mathrm{T}$

$15.4 \%$ meth

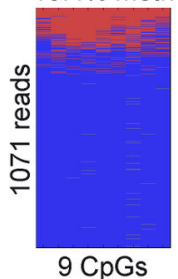

$\mathrm{G} / \mathrm{T}$

$65.6 \%$ meth

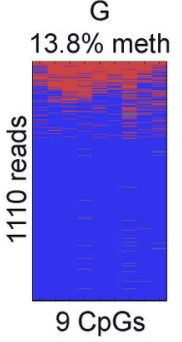

$\mathrm{T}$

13471

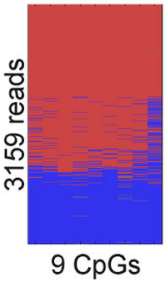

D
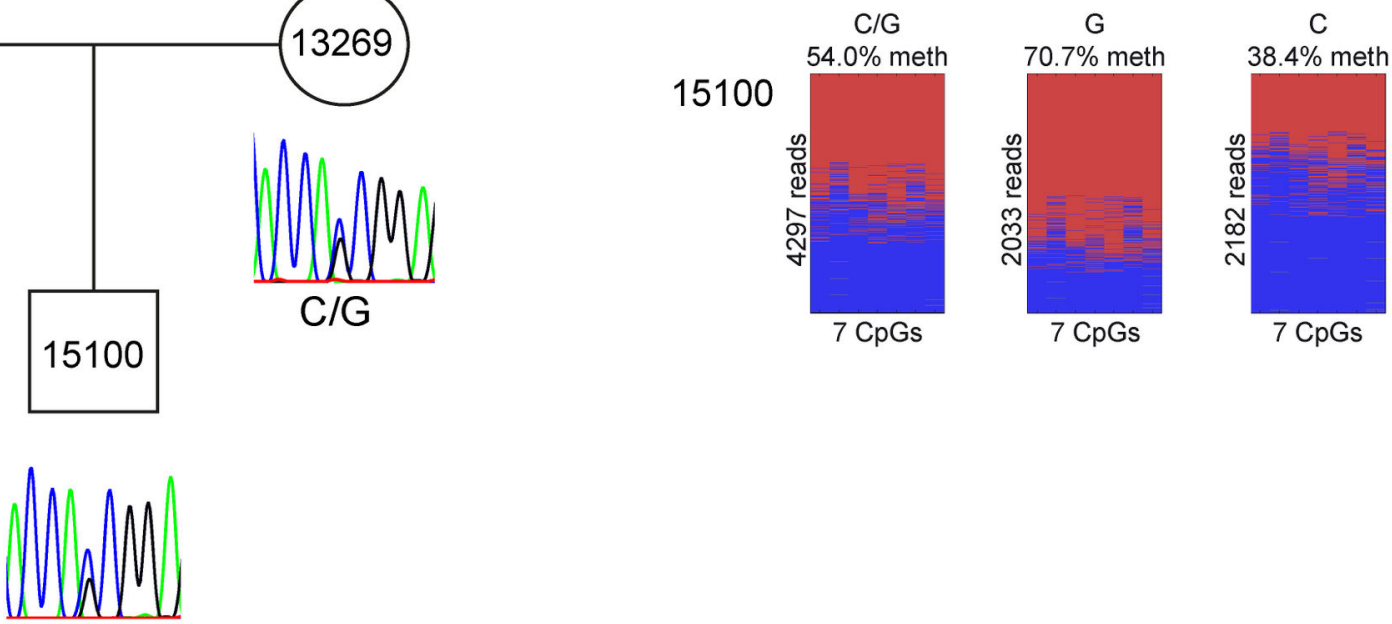
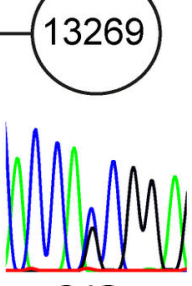

$\mathrm{C} / \mathrm{G}$

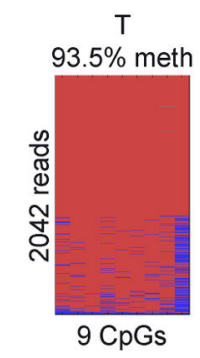

$\mathrm{C} / \mathrm{G}$

Figure 3. Analysis of parent-of-origin-specific methylation of the retrocopy in the $R B 1$ gene. (A) All rhesus macaque family members are heterozygous $(\mathrm{G} / \mathrm{T})$, thus, the parental origin of the alleles cannot be resolved. (B) One allele is methylated and the other allele is unmethylated in both individuals studied. (C) The offspring of the marmoset family carries an informative SNP (C/G). (D) The degree of methylation of the supposed maternal allele is $72 \%$, whereas the supposed paternal allele shows $39 \%$ methylation. A, C Sanger sequencing of genomic DNA; B, D deep bisulfite sequencing.

doi: 10.1371/journal.pone.0081502.g003

parental gene contains another CpG island (CpG 42 in the human). We analysed $15 \mathrm{CpGs}$ within human $\mathrm{CpG} 42$ and found full methylation (Figure 1, Figure S4).

As there are no $\mathrm{CpG}$ islands annotated in the first part of exon 4 of the retrocopies on chromosome 22 in human and chimpanzee, we analysed the methylation of the region corresponding to CpG 85 in human. We could not design specific primers for each of the four retrocopies on human chromosome 22 as they have a very high sequence identity. Therefore, we amplified all copies at once for the methylation analysis. We only obtained reads from methylated sequences (Figure 1, Figure 2). Methylation analysis of chimpanzee chromosome 22 retrocopies showed that the region corresponding to $\mathrm{CpG} 85$ in human is fully methylated in each 


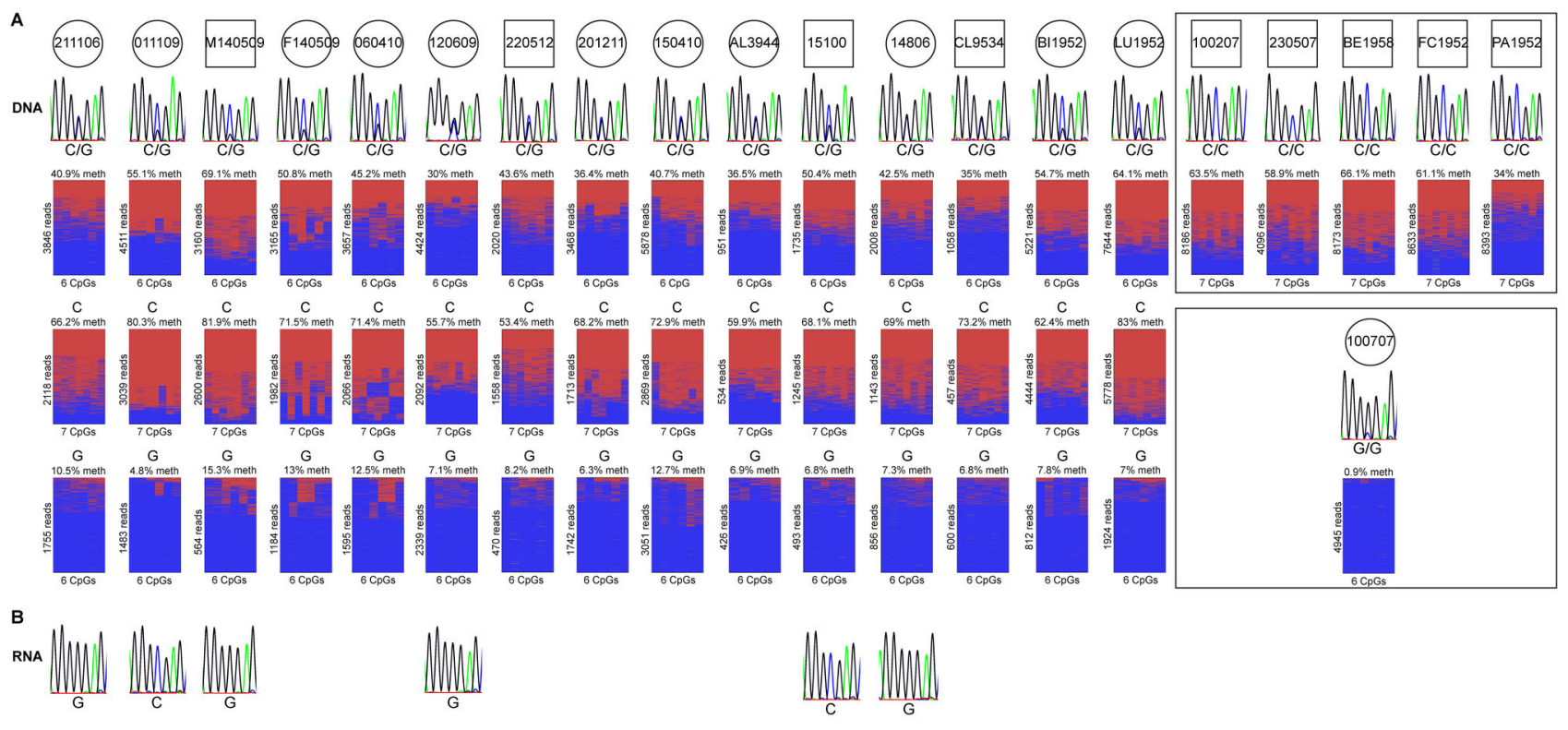

Figure 4. Allele-specific methylation and independent monoallelic expression of the retrocopy on chromosome 4 in marmoset. (A) A SNP (C/G) was used to distinguish the alleles. The mean methylation in the 15 heterozygous individuals was $30-69 \%$, the $\mathrm{G}$ allele showed $5-15 \%$ methylation and the $\mathrm{C}$ allele showed $53-83 \%$ methylation. The 5 individuals homozygous for the $C$ allele showed a mean methylation level of $34-66 \%$. The individual homozygous for the $G$ allele (100707) showed no methylation. Thus, the methylation pattern of the retrocopy on marmoset chromosome 4 is allele-specific. red, methylated; blue, unmethylated. (B) By RT-PCR and sequencing, a transcript specific for this retrocopy was identified in 6 heterozygous individuals (no RNA was available from the other heterozygous individuals). This transcript is monoallelically expressed and its expression is not regulated by DNA methylation as transcripts from methylated or unmethylated alleles were obtained.

doi: 10.1371/journal.pone.0081502.g004

retrocopy (Figure 1, Figure 2 and Figure S5). The same result was obtained for the chimpanzee retrocopy on chromosome 8 (Figure 1, Figure 2).

Analysis of the CpG island in exon 4 of the retrocopy on marmoset chromosome 4 showed reads from methylated and unmethylated sequences. We analysed samples from two individuals and obtained methylations levels of $61 \%$ and $50 \%$ (Figure 2). An informative SNP (C/G) was used to check whether methylation of this retrocopy is allele-specific and to distinguish alleles of 15 individuals (Figure 4A). The mean methylation level in these 15 individuals was $30-69 \%$. All $15 \mathrm{G}$ alleles present in these individuals were almost unmethylated (5-15\% methylation) and all $15 \mathrm{C}$ alleles showed a methylation level of $53-83 \%$ (Figure 4A). Five individuals homozygous for the $\mathrm{C}$ allele showed a methylation level of $34-66 \%$ and one individual homozygous for the $\mathrm{G}$ allele was unmethylated $(1 \%)$. Apparently, the methylation pattern of the retrocopy on marmoset chromosome 4 is allele-specific.

\section{The retrocopy on marmoset chromosome 4 is monoallelically transcribed}

We analysed marmoset chromosome 4 more closely by RT$\mathrm{PCR}$ and sequencing and identified a transcript specific for this retrocopy (Figure 4B, Figure S6). Furthermore, an informative SNP showed that this transcript is monoallelically expressed (Figure 4B, Figure S6). However, expression does not appear to be methylation-specific because transcripts were obtained from the methylated (2 individuals) or unmethylated (4 individuals) allele (no RNA was available from the other heterozygous individuals). Again, a statement on the parental origin of the expressed allele is not possible because of microchimerism in marmoset [12]. We conducted 3'-RACE and 5'-RACE to determine the transcription start site and the total length of the transcript detected by a non-intron-spanning RTPCR, but we did not obtain specific products.

\section{PPP1R26P1 serves as a promoter for an alternative $R B 1$ transcript in rhesus macaque}

As we have previously shown that an alternative transcript is expressed in humans [10], we here used RT-PCR and sequencing to determine if alternative $R B 1$ transcripts are expressed in primates other than humans and if expression is monoallelic. RNA from blood was available from a rhesus macaque family and a marmoset family with individuals heterozygous for a SNP in PPP1R26P1 (Figure 3A and C). Unfortunately, RT-PCR did not work on the RNAs from the rhesus macaque and marmoset individuals carrying the SNP. We could not detect expression of the 2B-transcript in marmoset although RT-PCRs with several different primers were tested. However, we obtained an RT-PCR product specific for the 2B-transcript for RNA from an uninformative rhesus macaque individual. Thus, the alternative $R B 1$ transcript 


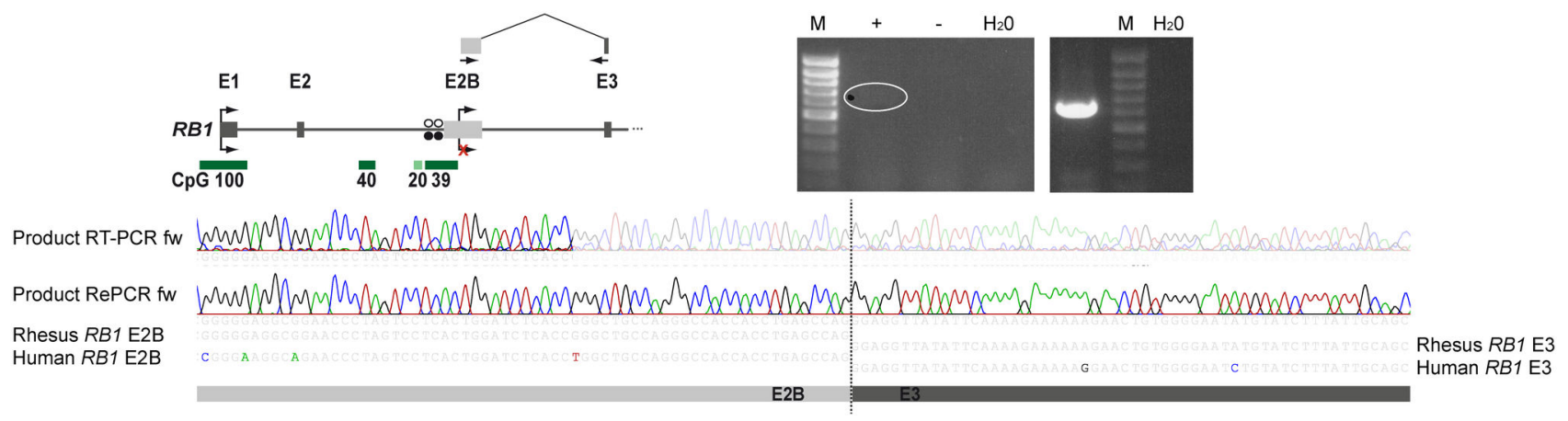

Figure 5. Expression of the alternative RB1 transcript in rhesus macaque. By exon-connection RT-PCR of CpG 39 and exon 3 of the rhesus macaque RB1 gene an alternative RB1 transcript was detected. Top left: scheme of rhesus macaque RB1 and position of the primers. Top right: PCR products of RT-PCR and RePCR (reamplification of the RT-PCR product); as there was only a faint band visible after RT-PCR (white circle) RePCR was performed. Sequence analysis of the RT-PCR and RePCR product showed that it is specific for the 2B-transcript (bottom). M, MassRuler low range - Thermo Scientific, +, mRNA with reverse transcriptase (RT); -, mRNA without $\mathrm{RT} ; \mathrm{H}_{2} \mathrm{O}$, negative control.

doi: 10.1371/journal.pone.0081502.g005

is present in rhesus macaque, but it remains unsolved whether it is parent-of-origin-specifically expressed (Figure 5).

\section{Discussion}

Imprinted expression of the human RB1 gene, a tumor suppressor gene with a plethora of functions [13], is the result of evolutionary change of a retrocopy of PPP1R26 that was inserted into intron 2 of the $R B 1$ gene [10]. The comparison of the sequences of PPP1R26 and its retrocopies in different primates performed in this study revealed that the PPP1R26 gene is present in members of Strepsirrhini (galago) and Haplorrhini (Anthropoidea and tarsier). PPP1R26P1, however, was detected in all Anthropoidea species analysed here but not in tarsier or galago. This suggests that retrotransposition of PPP1R26 is likely to have occurred before the split between Catarrhini and Platyrrhini. A further step in the evolution of PPP1R26P1 in Anthropoidea is an insertion of an $\sim 300$ bp Alu repeat between exon 3 and exon 4 of PPP1R26P1. This repeat is missing in marmoset, and thus, was transposed into this region after the split between Platyrrhini and Catarrhini. Also after this split, but restricted to the clade that includes the marmoset, is a duplication of an $\sim 550$ bp region of exon 4 of PPP1R26P1.

Imprinted expression of the human RB1 gene is linked to differential expression from a promoter within a differentially methylated $\mathrm{CpG}$ island, CpG 85, located in the part of $P P P 1 R 26 P 1$ that is derived from exon 4 of the parental gene. All species known to have PPP1R26P1 also have a CpG island that corresponds to $\mathrm{CpG} 85$ in human. It is likely that a CpGrich progenitor region of this $\mathrm{CpG}$ island was already present in the retrocopy that originally retrotransposed into the $R B 1$ gene, because in species with $P P P 1 R 26 P 1$ all extant orthologs of $P P P 1 R 26$ have one or two CpG islands in the 5'-part of exon 4, albeit of varying size. Large CpG islands (>300 bp) are also present in PPP1R26 retrocopies on chimpanzee chromosome 8 and marmoset chromosome 4 , further supporting that the parental PPP1R26 gene contained a $\mathrm{CpG}$ island in the 5'-part of exon 4.

In all primate species that we analysed, the CpG island in exon 4 of PPP1R26 is methylated (Figure 1 pie charts, Figure 2), making it prone to CpG-loss by deamination. But as this region is fully contained in the open reading frame of PPP1R26, purifying selection can contribute to stabilization of its CpG content. The mechanisms that stabilize the CpG content in the CpG island in PPP1R26P1 are less obvious. One possible factor is differential methylation of the human CpG 85 in PPP1R26P1 and its homologues in the other primate species tested here. We have no explanation why the orangutan shows differential methylation only at a few CpG sites in this CpG island. In the marmoset individual carrying a SNP $(C / G)$, the supposed maternal and paternal alleles showed a degree of methylation of $71 \%$ and $38 \%$, respectively. This does not reveal a clear parent-of-origin-specific methylation pattern, which might be due to mircochimerism [12]. Although all members of the analysed rhesus macaque family are heterozygous and the parental origin of the alleles remains unresolved, it is likely that the methylation in rhesus macaque is parent-of-origin-specific like in human, as the detected monoallelic methylation is not allele-specific (Figure 3). Furthermore, it is plausible that the analysed CpG island in rhesus macaque is paternally unmethylated and maternally methylated like in human. Regions with differentially methylated CpGs and lower methylation in the male germ line are under lower mutational pressure due to methylationcoupled deamination compared to regions with lower methylation in the female germ line [14]. Moreover, it has been shown that the rate of $\mathrm{CpG}$ loss through non-deamination substitutions is also lower than expected in differentially methylated regions that serve as imprinting control regions [15].

We have shown in human that the PPP1R26P1 region that was derived from exon 4 of PPP1R26 has evolved into a promoter and an initial exon of an alternative $R B 1$ transcript 
that is expressed from the unmethylated allele at CpG 85 [10]. This transcript was also detected in rhesus macaque, suggesting that PPP1R26P1 gained this function prior to the split of human and rhesus macaque. We attempted RT-PCR detection of this transcript in marmoset with different primers but obtained no product. We cannot exclude that an alternative $R B 1$ transcript is present in marmoset that we failed to detect for technical reasons only. Alternatively, such a transcript is not expressed in marmoset or at levels below the sensitivity of our approach.

The PPP1R26P1 in Catarrhini (human, chimpanzee, orangutan, rhesus macaque) have an insertion of an Alu repeat located 3' relative to the start and direction of the alternative transcript. This repeat is not present in marmoset PPP1R26P1. It has been shown that Alu sequences inserted in the vicinity of promoters can enhance transcription rates [16]. Moreover, this repeat may also contribute to parent-of-origin-specific methylation of $\mathrm{CpG} 85$ and its homologues, as Rubin and colleagues reported that Alu repeats are differentially methylated in primate germ cells [17]. They found hypomethylated and hypermethylated states in sperm and oocytes, respectively. This fits to our findings for the human Alu element in PPP1R26P1, which was completely unmethylated in sperm (Figure S7A). In blood however it showed a methylated state (Figure S7B). In the male germ line, Alu elements are selectively protected from DNA methylation by a specific Alu binding protein [18]. As hypomethylation in sperm does not affect all Alu sequences, there must be additional factors such as transcription, chromosomal location and nucleotide environment that may initialize and/or stabilize the methylation state $[17,19]$. Hellmann-Blumberg et al. showed that the methylation status of Alu elements varies in different tissues Alus hypomethylated in sperm were completely methylated in spleen, fitting to our findings in human blood (Figure S7B) [20]. It is conceivable that the Alu element contributes to the establishment of the imprint but not to its maintenance. Apparently, the Alu element becomes methylated during differentiation when the specific Alu binding protein that provides the protection against methylation is not present. Possibly once the imprint is set by the Alu element and transcription takes place, the state of the imprint is maintained by transcription or rather the binding of transcription factors.

Using a SNP, we could show that the methylation of the retrocopy on marmoset chromosome 4 , which consists of full exon 1-4 and no Alu insertion, is allele-specific. In this specific case microchimerism is irrelevant because the parental origin would not have an impact on DNA methylation. The marmoset homozygous for the $\mathrm{G}$ allele showed no methylation, whereas all marmosets homozygous for the C allele showed $34-66 \%$ methylation. Heterozygous marmosets had an almost unmethylated $G$ allele (5-15\% methylation) and 53-83\% methylation of the $\mathrm{C}$ allele. Expression analysis revealed a transcript specific for this retrocopy that is monoallelically expressed but not in a methylation-specific manner. Due to the possibility of microchimerism in marmosets it is not possible to say whether the expression is parent-of-origin-specific [12]. Thus, the retrocopy on chromosome 4 in marmoset has allelespecific methylation and is monoallelically expressed, but it is not imprinted. It appears that this particular locus shows random monoallelic expression. The region that includes the retrocopy on chromosome 4 in marmoset corresponds to human chromosome 6 and contains several genes of the immune system, such as $H L A-E$ and TRIM39. Several genes in human and mouse that are autosomal monoallelically expressed have functions in the immune or nervous system $[21,22,23]$. During differentiation, one allele is randomly silenced while the other allele remains active. This monoallelic state is then stably maintained across cell generations [24]. The expression observed at the retrocopy on chromosome 4 in marmoset could be an example for autosomal monoallelic expression, but it remains unclear whether this retrocopy has any contribution to this.

The retrocopies of PPP1R26 on chromosome 22 in human and chimpanzee are located intergenically. Assuming that the retrocopies had a $\mathrm{CpG}$ island at the time of insertion, all retrocopies underwent rapid decay of their $\mathrm{CpG}$ content and show full methylation at the $\mathrm{CpG}$ dinucleotides that remained. It appears that the genomic environment in which these retrocopies were placed provided no portal of entry for remodelling of gene regulation. In contrast, the retrocopies inserted in or near genes gained differential methylation and regulatory functions, which in turn recruited the evolutionary forces that helped to maintain their high CpG content. Thus, our data supports the concept that genomic imprinting is based on host defence mechanisms, and that the epigenetic fate of a PPP1R26 retrocopy after integration depends on the DNA sequence and selective forces at the integration site $[25,26,27,28]$.

\section{Materials \& Methods}

\section{Ethics statement}

Human blood samples from blood donors were obtained after written informed consent and were anonymised. The study was approved by the ethics committee of the University Duisburg-Essen (10-4396).

Non-human primate samples: Blood sampling procedures were conducted at the German Primate Centre in Göttingen. The studies were performed in accordance with the German Animal Welfare Act (Tierschutzgesetz der Bundesrepublik Deutschland 25.05.1998). This includes supervising and advice by the institutional animal welfare officer and approval by the governmental veterinary authorities. The corresponding reference number of the approval for blood sampling is 33.9-425-05-10A102 given by LAVES (Lower Saxony State Office for Consumer Protection and Food Safety). The ongoing of the procedures were controlled and supervised by the local and regional veterinary authorities, the veterinary staff and the animal welfare officer of the German Primate Centre. The animals are kept under conditions documented in the European Directive 2010/63/EU (directive on the protection of animals used for experimental and other scientific purposes) and the EU Recommendations 2007/526/EG (guidelines for the accommodation and care of animals used for experimental and other scientific purposes). These conditions are consistent with the regulations of the Guide for Care and Use of Laboratory 
Animals by the National Research Council (USA). The three Rs are considered using the 3Rs Guidelines for Primate Accommodation, Care and Use by the National Centre for the Replacement, Refinement and Reduction of Animals in Research (UK).

The blood samples were obtained in combination with the annual health monitoring tests of the German Primate Center breeding colonies or in combination with necessary veterinary procedures in stock animals. None of the animals were euthanized. The procedures were performed in accordance with the described regulations of the local and regional veterinary authorities and under attention of the national and European animal welfare regulations (EU directive 2010/63 EU, German Animal Welfare Act). The institutional animal welfare officer, who has to agree to the procedure, was informed prior to the blood withdrawal.

Below are details of housing conditions, enrichment and feeding.

Marmosets:

1 housing conditions

- indoor rooms with cages: 1qm and $2.50 \mathrm{~m}$ height for pairs and families with offspring; cages can be combined to allow larger groups

- indoor room: 14 to $18 \mathrm{qm}$, heated to $25^{\circ} \mathrm{C}$, humidity $60 \%$

- wood bedding on the ground

- enrichment with wood (bar, branches), ropes, sleeping boxes

2 feeding:

- two times per day in changing composition

- fruits, vegetables, special primate pellets, seeds

- cooked potatoes, eggs, rice, mealworms, grass hoppers, curd cheese, yoghurt

- Arabic gums

- water ad libitum

The German Primate Centre has a long standing experience in breeding, keeping and using non-human laboratory primates. Persons, who are carrying out procedures on animals, taking care for the animals or design the projects, have the authorisation by the veterinary authorities. An internal control system by the veterinary staff of the centre is established. This includes health monitoring, housing conditions, primate husbandry, care and environmental enrichment.

Additional non-human primate samples: Marmoset (Callithrix jacchus) DNA was obtained from blood samples provided by Stefan Schlatt, Centre of Reproductive Medicine and Andrology, Muenster. The blood samples were drawn related to experiments approved by the relevant authorities in accordance with the German Federal Law on the Care and Use of Laboratory Animals (LANUV North Rhine-Westphalia, Licence No. 84-02.05.20.12.0.18). The marmoset monkeys from our breeding colony were kept in pairs/families under a 12 $\mathrm{h}$ light: $12 \mathrm{~h}$ darkness regimen and fed food pellets from Altromin (Lage, Germany) composed for marmosets together with beef or chicken meat and a daily supplement of fresh fruits and vegetables. They had unlimited access to tap water. Housing and exercise conditions were identical for all animals. No animals were sacrificed in the frame of this study. Blood samples $(0.2 \mathrm{ml})$ were taken from the Vena femoralis. Sampling was performed without sedation by manual fixation by experienced animal caretakers, a method approved by the local authorities and proved to be less stress-causing than prior sedation of animals. Every animal was rewarded flour worms after blood sampling. The LANUV North Rhine-Westphalia, Licence No. 84-02.05.20.12.0.18 refers to the approval for blood sampling by the Ministry of Environment of North RhineWestphalia, Germany.

\section{In silico analysis}

The BLAT tool of the UCSC genome browser was used for in silico analyses (human - Homo sapiens, hg19; chimpanzee Pan troglodytes, panTro4; orangutan - Pongo abelii, ponAbe2; rhesus macaque - Macaca mulatta, rheMac3; marmoset Callithrix jacchus, calJac3; tarsier - Tarsius syrichta, tarSyr1; galago - Otolemur garnettii, otoGar3). We also performed blastn which has more relaxed parameters and still ended up with no retrocopy of PPP1R26 in galago or tarsier. The query sequence used for all searches was the mRNA sequence of PPP1R26 (NM_014811). We also used Progressive Mauve to align the intron 2 regions of $R B 1$ of tarsier, galago, human and marmoset and again obtained the same results.

\section{Bisulfite treatment}

Bisulfite treatment was carried out using the EZ DNA Methylation-Gold Kit (Zymo Research Europe, Freiburg, Germany) according to the manufacturer's protocol.

\section{Deep bisulfite sequencing}

Generation of bisulfite amplicon libraries, sample preparation and sequencing on the Roche 454 GS junior system was carried out as previously described [29]. Primer sequences are given in Table S1.

For data analysis, we used the Python-based amplikyzer software developed in-house [unpublished, available under the open source MIT License at https://code.google.com/p/ amplikyzer/ ].

\section{Genomic sequence analysis}

Primers designed for $R B 1$ (rhesus macaque, marmoset) and PPP1R26P1 on marmoset chromosome 4 are listed in Table S1. For each PCR, $100 \mathrm{ng}$ genomic DNA was used in a total volume of $25 \mu \mathrm{l}$. PCR conditions were as follows (for $\mathrm{Tm}=\mathrm{X}$ see Table S1): $95^{\circ} \mathrm{C}$ for $10 \mathrm{~min} ; 35$ cycles of $95^{\circ} \mathrm{C}$ for $20 \mathrm{~s}, \mathrm{X}^{\circ} \mathrm{C}$ for $30 \mathrm{~s}, 72^{\circ} \mathrm{C}$ for $1 \mathrm{~min} ; 72^{\circ} \mathrm{C}$ for $7 \mathrm{~min}$. The PCR products were purified by MultiScreen Filtration (Millipore, Billerica, MA, USA). The sequence reactions were performed with Big Dye Terminators (BigDye Terminator v1.1 Cycle Sequencing Kit, Applied Biosystems, Foster City, CA, USA) and the cycle sequencing procedure. Reaction products were analysed with an ABI 3100 automatic capillary Genetic Analyzer and Sequencing Analysis software (Applied Biosystems, Foster City, CA, USA). 


\section{Expression analysis}

RT-PCRs were performed with the GeneAmp RNA PCR Kit (Applied Biosystems, Foster City, CA, USA). Total RNA from blood was reverse transcribed with random hexamers. For amplification, the Advantage cDNA Polymerase Mix (Clontech, Mountain View, CA, USA) and the Phusion Flash High Fidelity PCR Master Mix (Finnzymes, Espoo, Finland) were used. PCR products were checked on an agarose gel and purified by gel extraction (Wizard SV Gel and PCR Clean-Up System, Promega). The primers used for the different RT-PCRs are listed in Table S1. For exon connection PCR at the RB1 locus in rhesus macaque, we designed primers where the forward primer anneals to the CpG $39 \mathrm{CpG}$ island in the retrocopy in $R B 1$ intron 2 and the reverse primer anneals to exon 3 of the RB1 gene. For amplification, we used the Phusion Flash High Fidelity PCR Master Mix (Finnzymes, Espoo, Finland). PCR conditions were as follows: $98^{\circ} \mathrm{C}$ for $10 \mathrm{sec} ; 35$ cycles of $98^{\circ} \mathrm{C}$ for $1 \mathrm{sec}, 61^{\circ} \mathrm{C}$ for $5 \mathrm{sec}, 72^{\circ} \mathrm{C}$ for $15 \mathrm{sec} ; 2^{\circ} \mathrm{C}$ for $1 \mathrm{~min}$.

For the expression analysis at the retrocopy on chromosome 4 in the marmoset we designed primers located in the analysed CpG island and used the Advantage cDNA Polymerase Mix (Clontech, Mountain View, CA, USA) for amplification. PCR conditions were as follows: $95^{\circ} \mathrm{C}$ for $1 \mathrm{~min} ; 5$ cycles of $95^{\circ} \mathrm{C}$ for $20 \mathrm{sec}, 68^{\circ} \mathrm{C}$ for $3 \mathrm{~min} ; 35$ cycles of $95^{\circ} \mathrm{C}$ for $20 \mathrm{sec}, 66^{\circ} \mathrm{C}$ for 3 min; 5 cycles of $95^{\circ} \mathrm{C}$ for $20 \mathrm{sec}, 60^{\circ} \mathrm{C}$ for $3 \mathrm{~min} ; 68^{\circ} \mathrm{C}$ for 3 $\min$.

\section{Supporting Information}

Table S1. Primer sequences. (DOCX)

Figure S1. Quantitative PCR analysis of PPP1R26P1 in orangutan. The normalized ratios of the target locus and the reference locus for each sample are shown (error bars = normalized ratio error; for the analysis the LightCycler 480 Software was used). TRPS1 was used as a reference locus and rhesus macaque was used as a reference genome as it only contains one PPP1R26P1 copy. The data show that there is only one copy of PPP1R26P1 present in the orangutan genome. Rhe 42, Rhe 46, Rhe 48 - rhesus macaque samples; OU 518, OU 519 - orangutan samples.

(TIF)

Figure S2. Progressive Mauve alignments. In the upper part the alignment of the human $R B 1$ intron 2 region and the tarsier $R B 1$ intron 2 region is shown. Below the alignment of the human $R B 1$ intron 2 region and the Otolemur RB1 intron 2 region is shown. For both tarsier and Otolemur the neighbouring sequences of PPP1R26P1 are present but PPP1R26P1 itself is clearly absent. Human hg19, chr13:48880916-48917350_plus strand; tarsier syrTar1, scaffold_292:1-45070_minus strand; Otolemur otoGar3, GL873625:966173-991826_plus strand.

(TIF)
Figure S3. Methylation status of CpG 81 in orangutan PPP1R26P1. The alleles for individual 519 were separated using a SNP $(\mathrm{C} / \mathrm{G})$. The degree of methylation for each $\mathrm{CpG}$ site is shown. On the left: alleles, on the right: mean methylation over the four $\mathrm{CpG}$ sites and number of reads.

(TIF)

Figure S4. Methylation status of CpG 42 in human PPP1R26P1. All analysed samples are fully methylated. red, methylated; blue, unmethylated. Blood sample IDs are given under the images (C1, C2 and C3 - human).

(TIF)

Figure S5. Methylation status of the three retrocopies on chimpanzee chromosome 22. All three retrocopies on chimpanzee chromosome 22 are methylated. red, methylated; blue, unmethylated. Samples IDs are given under the images (1014, 1126 - chimpanzee).

(TIF)

Figure S6. Methylation and expression analysis of the retrocopy on marmoset chromosome 4. A SNP (C/G) was used to distinguish the alleles. In all analysed samples, the $G$ allele showed $7 \%$ methylation and the $C$ allele showed $56-68 \%$ methylation. The individual homozygous for the $G$ allele (100707) showed no methylation. Thus, the methylation pattern of the retrocopy on marmoset chromosome 4 is allele-specific. red, methylated; blue, unmethylated. By RT-PCR and sequencing, a transcript specific for this retrocopy was identified. Based on the informative SNP $(\mathrm{C} / \mathrm{G})$, it could be shown that this transcript is monoallelically expressed. Moreover, the expression is not regulated by DNA methylation as transcripts from methylated or unmethylated alleles were obtained.

(TIF)

Figure S7. Methylation status of the Alu element in PPP1R26P1 of human sperm. (A) The Alu element is completely unmethylated in sperm (1168-4 and 1167-33 human) and (B) methylated in blood ( $\mathrm{C} 1, \mathrm{C} 2$ - human). red, methylated; blue, unmethylated.

(TIF)

\section{Acknowledgements}

We thank Laura Steenpass for helpful discussion, Katrin Rademacher for bioinformatical help, Nicholas Wagner for helping editing the manuscript, Melanie Heitmann for expert technical assistance and Tamara Becker for taking blood samples from marmosets.

\section{Author Contributions}

Conceived and designed the experiments: DK KB DL $B H$. Performed the experiments: DK SK. Analyzed the data: DK KB. Contributed reagents/materials/analysis tools: CR JG. Wrote the manuscript: DK DL. Revised and approved the manuscript: DK KB CR JG SK BH DL. 


\section{References}

1. Kaessmann H, Vinckenbosch N, Long M (2009) RNA-based gene duplication: mechanistic and evolutionary insights. Nat Rev Genet 10: 19-31. doi:10.1038/ni0109-19. PubMed: 19030023.

2. Betrán E, Wang W, Jin L, Long M (2002) Evolution of the phosphoglycerate mutase processed gene in human and chimpanzee revealing the origin of a new primate gene. Mol Biol Evol 19: 654-663. doi:10.1093/oxfordjournals.molbev.a004124. PubMed: 11961099.

3. Poliseno L, Salmena L, Zhang J, Carver B, Haveman WJ et al. (2010) A coding-independent function of gene and pseudogene mRNAs regulates tumour biology. Nature 465: 1033-1038. doi:10.1038/ nature09144. PubMed: 20577206

4. Tam OH, Aravin AA, Stein P, Girard A, Murchison EP et al. (2008) Pseudogene-derived small interfering RNAs regulate gene expression in mouse oocytes. Nature 453: 534-538. doi:10.1038/nature06904. PubMed: 18404147.

5. Korneev SA, Park JH, O'Shea M (1999) Neuronal expression of neural nitric oxide synthase (nNOS) protein is suppressed by an antisense RNA transcribed from an NOS pseudogene. J Neurosci 19: 7711-7720. PubMed: 10479675.

6. Wood AJ, Schulz R, Woodfine K, Koltowska K, Beechey CV et al. (2008) Regulation of alternative polyadenylation by genomic imprinting Genes Dev 22: 1141-1146. doi:10.1101/gad.473408. PubMed: 18451104.

7. Cowley M, Wood AJ, Böhm S, Schulz R, Oakey RJ (2012) Epigenetic control of alternative mRNA processing at the imprinted Herc3/Nap115 locus. Nucleic Acids Res 40: 8917-8926. doi:10.1093/nar/gks654. PubMed: 22790983.

8. Wang $Y$, Joh K, Masuko S, Yatsuki H, Soejima H et al. (2004) The mouse Murr1 gene is imprinted in the adult brain, presumably due to transcriptional interference by the antisense-oriented U2af1-rs1 gene. Mol Cell Biol 24: 270-279. doi:10.1128/MCB.24.1.270-279.2004. PubMed: 14673161.

9. Whitelaw E, Martin DI (2001) Retrotransposons as epigenetic mediators of phenotypic variation in mammals. Nat Genet 27: 361-365. doi:10.1038/86850. PubMed: 11279513.

10. Kanber D, Berulava T, Ammerpohl O, Mitter D, Richter J et al. (2009) The human retinoblastoma gene is imprinted. PLOS Genet 5: e1000790.

11. Gardiner-Garden M, Frommer M (1987) CpG islands in vertebrate genomes. J Mol Biol 196: 261-282. doi: 10.1016/0022-2836(87)90689-9. PubMed: 3656447.

12. Ross CN, French JA, Ortí G (2007) Germ-line chimerism and paternal care in marmosets (Callithrix kuhlii). Proc Natl Acad Sci U S A 104: 6278-6282. doi:10.1073/pnas.0607426104. PubMed: 17389380.

13. Dick FA, Rubin SM (2013) Molecular mechanisms underlying RB protein function. Nat Rev Mol Cell Biol 14: 297-306. doi:10.1038/ nrm3567. PubMed: 23594950.

14. Schulz R, Proudhon C, Bestor TH, Woodfine K, Lin CS et al. (2010) The parental non-equivalence of imprinting control regions during mammalian development and evolution. PLoS Genet 6: e1001214. PubMed: 21124941.

15. Cohen NM, Kenigsberg E, Tanay A (2011) Primate CpG islands are maintained by heterogeneous evolutionary regimes involving minimal selection. Cell 145: 773-786. doi:10.1016/j.cell.2011.04.024. PubMed: 21620139.

16. Ichiyanagi $\mathrm{K}$ (2013) Epigenetic regulation of transcription and possible functions of mammalian short interspersed elements, SINEs. Genes Genet Syst 88: 19-29. PubMed: 23676707.

17. Rubin CM, VandeVoort CA, Teplitz RL, Schmid CW (1994) Alu repeated DNAs are differentially methylated in primate germ cells. Nucleic Acids Res 22: 5121-5127. doi:10.1093/nar/22.23.5121. PubMed: 7800508.

18. Chesnokov IN, Schmid CW (1995) Specific Alu binding protein from human sperm chromatin prevents DNA methylation. J Biol Chem 270: 18539-18542. doi:10.1074/jbc.270.31.18539. PubMed: 7629183

19. Chotalia M, Smallwood SA, Ruf N, Dawson C, Lucifero D et al. (2009) Transcription is required for establishment of germline methylation marks at imprinted genes. Genes Dev 23: 105-117. doi:10.1101/gad. 495809. PubMed: 19136628.

20. Hellmann-Blumberg U, Hintz MF, Gatewood JM, Schmid CW (1993) Developmental differences in methylation of human Alu repeats. Mol Cell Biol 13: 4523-4530. PubMed: 8336699.

21. Gimelbrant A, Hutchinson JN, Thompson BR, Chess A (2007) Widespread monoallelic expression on human autosomes. Science 318: 1136-1140. doi:10.1126/science.1148910. PubMed: 18006746.

22. Zwemer LM, Zak A, Thompson BR, Kirby A, Daly MJ et al. (2012) Autosomal monoallelic expression in the mouse. Genome Biol 13: R10. doi:10.1186/gb-2012-13-2-r10. PubMed: 22348269.

23. Chess $A$ (2012) Mechanisms and consequences of widespread random monoallelic expression. Nat Rev Genet 13: 421-428. doi:10.1038/ni. 2285. PubMed: 22585065

24. Gimelbrant AA, Ensminger AW, Qi P, Zucker J, Chess A (2005) Monoallelic expression and asynchronous replication of p120 catenin in mouse and human cells. J Biol Chem 280: 1354-1359. PubMed: 15522875.

25. Barlow DP (1993) Methylation and imprinting: from host defense to gene regulation? Science 260: 309-310. doi:10.1126/science.8469984. PubMed: 8469984.

26. Suzuki S, Ono R, Narita T, Pask AJ, Shaw G et al. (2007) Retrotransposon silencing by DNA methylation can drive mammalian genomic imprinting. PLoS Genet 3: e55. doi:10.1371/journal.pgen. 0030055 . PubMed: 17432937.

27. Pask AJ, Papenfuss AT, Ager El, McColl KA, Speed TP et al. (2009) Analysis of the platypus genome suggests a transposon origin for mammalian imprinting. Genome Biol 10: R1. doi:10.1186/ gb-2009-10-1-r1. PubMed: 19121219.

28. Yoder JA, Walsh CP, Bestor TH (1997) Cytosine methylation and the ecology of intragenomic parasites. Trends Genet 13: 335-340. doi: 10.1016/S0168-9525(97)01181-5. PubMed: 9260521.

29. Beygo J, Citro V, Sparago A, De Crescenzo A, Cerrato F et al. (2013) The molecular function and clinical phenotype of partial deletions of the IGF2/H19 imprinting control region depends on the spatial arrangement of the remaining CTCF-binding sites. Hum Mol Genet 22: 544-557. doi: 10.1093/hmg/dds465. PubMed: 23118352. 